\title{
Potential, Practicalities and Problems of Aberration Minimized FESEM
}

\author{
Edward D Boyes
}

DuPont Company, CR\&D, Experimental Station, PO Box 80356-383, Wilmington, DE 19880-0356

An advantage of SEM is the convenient bulk specimen form, including unthinned silicon wafers. No sample : no data. Historically there have been rather low performance expectations for this type of microscope; based in part on a limited, or actually incorrect, understanding of the beam-specimen interaction physics. However, it now appears the spatial resolution of secondary electron imaging at low beam energies (where most action is these days) is limited primarily by the instrument probe size; convoluted in some cases (as BSE, X-rays) with the signal generation range in the sample [1]. Even the latter is greatly reduced at low beam energies. Fig.1 shows images of carbon nanotubes obtained from more or less the same area at $30 \mathrm{kV}$ and $1.5 \mathrm{kV}$ at $250,000 \mathrm{x}$. No features detected at $30 \mathrm{kV}$ are unresolved at $1.5 \mathrm{kV}$; although, of course, the nature of the respectively quasi-transmission and surface sensitive images is quite different. In politically correct terms they are complementary. The smallest features, which may be SWNTs, have an increased image width at $1.5 \mathrm{kV}$ due to the inferior $<2 \mathrm{~nm}$ vs $<0.5 \mathrm{~nm}$ probe size under the respective conditions. The basic $30 \mathrm{kV}$ resolution, which is undoubtedly $<0.5 \mathrm{~nm}$ by the usual definitions, is more clearly indicated in Fig. 2 of Pt on C at 1,000,000x original magnification. There seems no good reason why the images at low voltages could not have a similar crisp resolution of $0.5 \mathrm{~nm}$ or less; except for the ballooning probe size of the instruments under these informative surface sensitive but challenging conditions.

The conclusion from this line of argument is that there is every reason to try to improve the probe size performance of the instrument. Previous work [1] has shown for many applications this is most important at low beam energies at or below $1.5 \mathrm{kV}$. Three approaches can be followed. Firstly, there is the prospect of lenses with lower natural aberrations, especially for operations only at low voltages, secondly, for SEM those lenses can be operated to minimize aberrations, and thirdly, at some point we need to move to active aberration correction [2]. To date the best images, with resolutions approaching $1 \mathrm{~nm}$ at $1-2 \mathrm{kV}$ and magnifications of $2-400,000 \mathrm{x}$, are probably still being produced with aberration minimized systems; new application data from which will be presented. However, with active correction it should be possible to design high resolution systems with greater operational flexibility, including a full range of tilting and complementary EDX multispectral nanochemical analysis - a particular strength of the SEM and other EMs. It should then be possible to resolve towards $0.5 \mathrm{~nm}$ at $1 \mathrm{kV}$, and extend high performance to $0.5 \mathrm{kV}$ or less using electrostatic beam retardation; avoiding the need to make a corrector work over a wider voltage range, especially if this is extended into the exciting very low voltage $(<0.1 \mathrm{kV}$ and even $<0.01 \mathrm{kV})$ regime.

Any and all of the above courses of action to improve image resolution depend on the rest of the stabilities in the instrument being adequate to support enhanced performance. In many SEMs, stage and other stabilities are already a limitation, but this can be gotten around by co-opting proven TEM technology. A very low AC field environment superior to that for a $1 \AA$ STEM is essential, if necessary with active compensation, but otherwise importing established engineering from the best STEM practices should prove effective. Mechanical and thermal stability needs are not quite so high, but the need for a clean near UHV vacuum (and sample) can only increase with an extended resolution requirement. This raises questions about how the sample is prepared and transferred. 
A fundamental problem in extended resolution SEM at low beam energies is the increased electron wavelength. To avoid a diffraction limited probe the aperture angle must be opened up and this has the ancillary effect of significantly reducing the available depth of field for sharp focus, which has previously been a hallmark of SEM. Fig. 3 shows some preliminary simple model estimations.

For high resolution FESTEM at or above $100 \mathrm{kV}$ the dominant aberration is spherical, but in many SEM applications the limitation is chromatic. In the case of our highly modified ultrahigh resolution FESEM [3] both $\mathrm{Cc}$ and $\mathrm{Cs}$ can be reduced to small fractions of a millimeter. Below $0.2 \mathrm{~mm}$ and $0.1 \mathrm{~mm}$ respectively are projected as being possible and 0.3 and $0.2 \mathrm{~mm}$, with EDX and some tilt, are now routine; and there is a better balance between the terms. In this system the spherical, chromatic and diffraction contributions are all important. The narrow energy spread of a cold field emission gun contributes to better performance, at least with modest beam currents, and the supposed stability issues can be solved with good vacuum design and aggressive processing. The scientific impact to be expected from new SEMs at or below $1 \mathrm{~nm}$ resolution at $1 \mathrm{kV}$ is exciting.
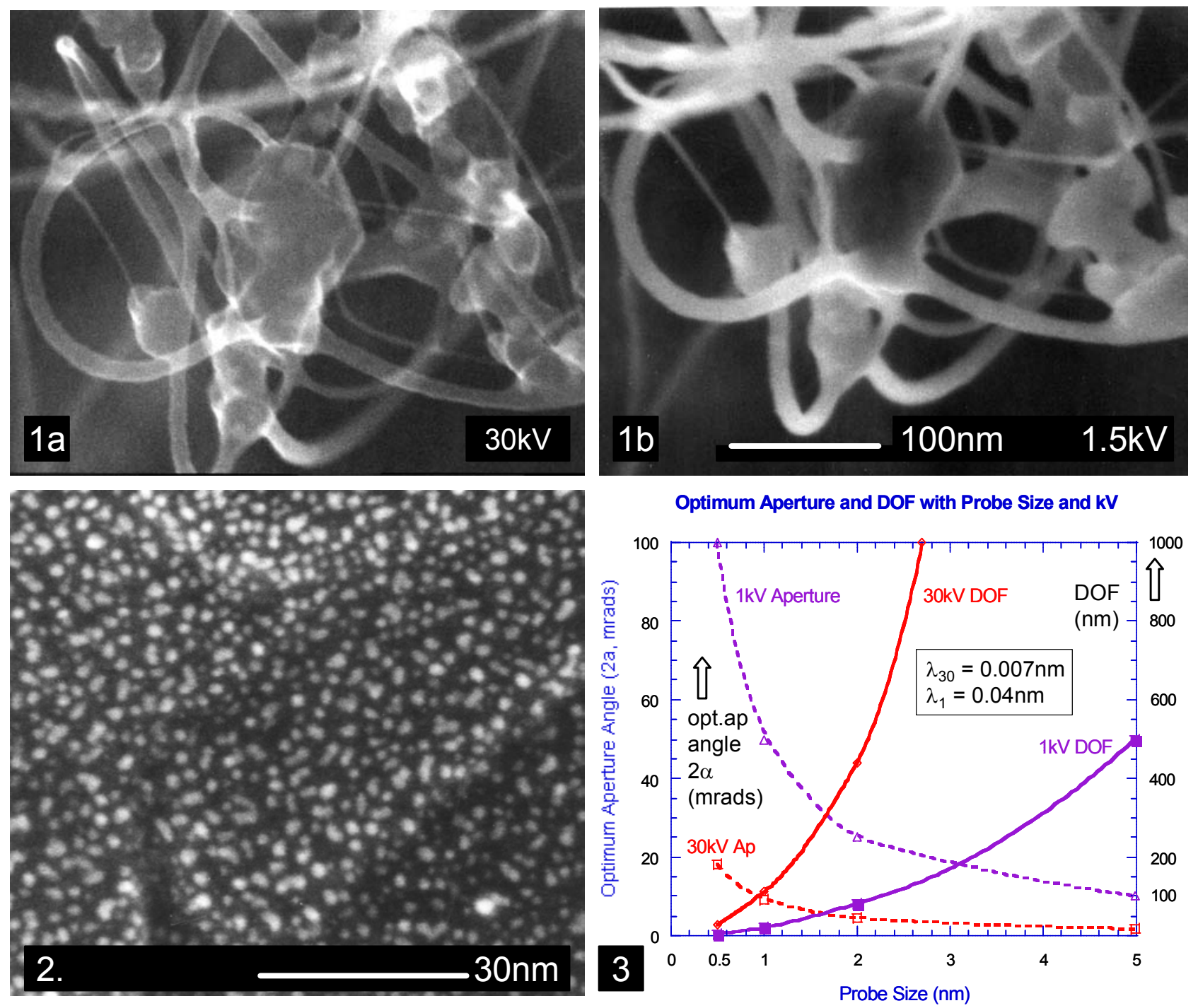

[1]. E D Boyes, Microscopy and Microanalysis, 6 (2000) 307

[2]. M Haider et al, Nature, 393 (1998) 768

[3]. E D Boyes, Proc EMC 2004, Belgian Society for Microscopy, Liege, 1 (2004) 319 